\title{
Expansión de la producción de aceite de palma en territorios campesinos, el caso de Palenque, Chiapas (1996-2018)
}

\section{Expansion of palm oil production in peasant territories, the case of Palenque, Chiapas (1996-2018)}

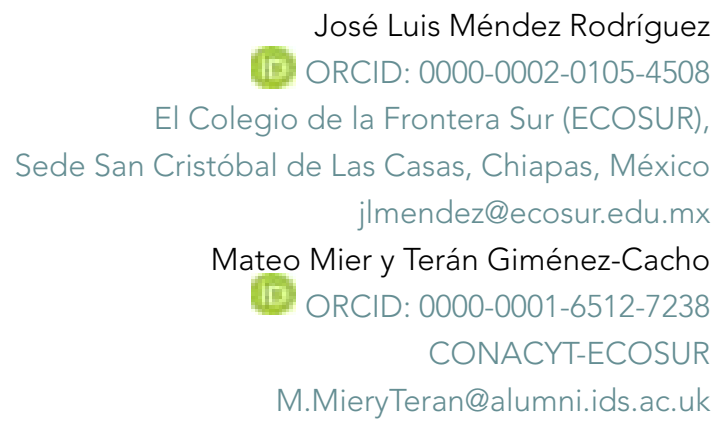

Resumen

Este artículo describe y analiza cómo la producción agroindustrial de aceite de palma se ha expandido en territorios campesinos del municipio de Palenque, Chiapas, México. El uso de un enfoque desde la ecología política empírica y cualitativa permitió centrarnos en los campesinos-palmeros y en sus relaciones con otros actores. Durante el trabajo de campo se realizaron entrevistas en plantaciones, localidades rurales y en la ciudad de Palenque. Los hallazgos indican que, en la zona recorrida, se está gestando una territorialidad expresada en la dependencia de los campesinos-palmeros a los ingresos monetarios de la venta del fruto de la palma, quienes en su mayoría no obtienen ganancias económicas considerables, pero sí las mínimas necesarias para mantenerse en la cadena de producción.

Palabras clave: ecología política, palma aceitera, territorialización, agroindustria, plantaciones comerciales.

Abstract

This paper describes and analyzes how agro-industrial palm oil production has expanded in small-scale agricultural territories pertaining to the municipality of Palenque, Chiapas State, Mexico. From the perspective of political ecology, using both empirical and qualitative studies, it was possible to focus on small-scale palm producers and their relationships with other stakeholders. During the fieldwork, interviews were carried out on plantations, rural sites, and the town of Palenque. Findings indicate that territoriality is emerging in the area studied that expresses the degree to which small-scale palm producers rely on income from the sale of oil palm fruit. Despite the fact that the income is not considerable, it suffices minimally in order to maintain the chain of production.

Keywords: political ecology, oil palm, territorialization, agroindustry, commercial plantations. 
Recibido: 10/09/2019

Aceptado: 19/06/2020

Publicado: 30/10/2020

\section{Introducción}

a expansión del cultivo de palma aceitera (Elaeis guineensis Jacq.) a escala agroindustrial ha modificado diversos paisajes y territorios agrícolas en los trópicos de Asia, África, Oceanía y América, en donde la implementación poco regulada de este cultivo ha implicado una larga sucesión de afectaciones sociales y ambientales, en particular en Malasia, Indonesia, Colombia, Guatemala y Brasil (Escobar, 2010; Bissonnette, 2012; Cajas-Castillo et al., 2015; Ávila y Albuquerque, 2018). Aunque esto se puede tomar como un indicador de precaución, no necesariamente nos explica el escenario resultante en otros territorios.

En una escala global, este cultivo se ha expandido bajo el impulso principal de agroindustrias, de Estados-nación y de organismos internacionales como el Banco Mundial y el Banco Interamericano de Desarrollo (BID), con la finalidad de aprovechar los aceites de sus frutos y semillas. Las empresas que producen alimentos procesados, cosméticos, jabones y detergentes, artículos farmacéuticos y algunos domésticos, impulsan una industrialización que demanda cada vez más la materia prima derivada de las plantaciones de palma aceitera; demanda que, según un reporte del Banco Mundial, se asocia al abandono de los productos derivados del petróleo (The World Bank, 2011). Si los requerimientos de estas industrias aumentan es de esperarse que soliciten mayor producción de palma.

Diversas investigaciones académicas en los últimos diez años (2009-2019) presentan cómo este cultivo se ha expandido en regiones específicas del sureste mexicano, particularmente en Chiapas. En las publicaciones de Castellanos (2015; 2018), Fletes y Bonnano (2015), Ávila et al. (2017), y Linares et al. (2018) se ha identificado al Estado mexicano como impulsor importante del establecimiento de plantaciones de palma aceitera. Dichos estudios han abordado los casos de la Costa-Soconusco y la región de Marqués de Comillas; excepto Linares (2018), quien se enfocó en el Valle de Tulijá, al sur de Palenque, esta última es una zona donde la expansión del cultivo de palma es más reciente (a partir de 1996). 
Este artículo presenta una explicación del caso de la expansión de este cultivo en territorios campesinos de Palenque, ubicado en el norte de Chiapas y colindante con el estado de Tabasco. La pregunta que nos interesa responder es: ¿Cómo la expansión de la palma aceitera ha generado cambios en las configuraciones territoriales de los campesinos de Palenque? Nuestro objetivo es identificar las tendencias más sobresalientes respecto a la actual reestructuración territorial de la región de Palenque a partir de la expansión del modelo agroindustrial de producción del aceite de palma. Exponemos que la expansión de cultivo de palma se expresa en: a) acciones concretas de promoción del cultivo por parte del Estado; b) tensiones entre la agroindustria de aceite y el campesinado; c) una valoración diferenciada de los efectos sociales de la palma por los mismos campesinos-palmeros; d) negociación entre agentes externos e internos; ${ }^{1}$ e) la relativa adaptabilidad de la actividad agroindustrial de palma aceitera en los territorios agrícolas de Palenque; y f) la emergencia de un hibridismo cultural entre lo campesino y lo agroindustrial (lo cual nos permite hablar de un campesino-palmero).

En las siguientes páginas ahondaremos en los aspectos que ayudan a entender por qué los campesinos-palmeros cultivan palma aceitera, cómo aconteció la llegada de este cultivo a Palenque, cómo es percibida actualmente la producción entre quienes sí cultivan la palma y entre quienes no lo hacen, y cómo es que, en general, la producción de aceite de palma está territorializándose en Palenque. Previamente presentamos el marco teórico que permitió analizar la territorialidad desde la ecología política, seguido de una sección metodológica.

\section{Ecología política y estudios sobre territorialidad}

El campo teórico de la ecología política tiene un carácter interdisciplinario y en las recientes décadas ha centrado su atención en demostrar:

cómo se forman las ideas y comprensiones sobre la naturaleza, y sobre la interacción de las personas con ella [... para] entender la forma en la que las interpretaciones limitan y dirigen lo que se considera cierto y deseable en relación al entorno, legitimando ciertas explicaciones y marginalizando otras (Robbins 2005, en Durand et al., 2011:289).

1 Esto resuena en lo que Rangel y Fletes (2010) denominan "guerras por el espacio», es decir, las continuas disputas y negociaciones para el uso del espacio agrícola. 
Además, dentro de la ecología política se retoman debates de larga data en otras disciplinas, generando estudios que centran la atención en documentar las luchas por el acceso y la apropiación de los recursos donde se ve involucrada una gran diversidad de actores sociales, y donde cobran relevancia las relaciones desiguales de poder y dominación (Durand et al., 2011:288). Esta y otras referencias de la ecología política ${ }^{2}$ han aportado a esta investigación para: identificar al campesinado como el principal sujeto colectivo de estudio; asumir una postura conceptual y teórica en torno al territorio como un espacio constituyente de las relaciones sociales que buscábamos observar y analizar; conocer acerca de la historia reciente de la región en cuanto a lo ambiental, lo agrario, lo agrícola y lo social; y hacer énfasis en la importancia de explicitar que se busca trascender los límites teóricos que dividen sociedad y naturaleza. La ecología política nos permitió enfocar la atención en los campesinos-palmeros (refiriéndonos a campesinos que producen palma), ${ }^{3}$ y estudiar las relaciones de este sujeto social con los actores que, argumentamos, dirigen el rumbo de la expansión de la palma. De entre los múltiples actores, como describiremos más adelante, destacan el Estado mexicano, las empresas y las organizaciones de productores.

Desde el campo teórico de la ecología política fue posible abordar la complejidad de la palma sin reducirla a una problemática productiva o ambiental únicamente. Propuesta ya hecha en los trabajos de Arturo Escobar (2010) en Colombia y de Antonio Castellanos (2015; 2018) en México. Escobar (2010) describe la expansión de la agricultura capitalista que, a través de la expansión de monocultivos de palma aceitera, se ha articulado territorialmente (en el Pacífico colombiano) lo que ha generado singulares expresiones de conflicto social:

La expansión de la palma de aceite se efectúa por la fuerza a través de la violencia y el desplazamiento; de hecho, junto con el cultivo de la coca, la palma de aceite se ha vuelto la razón principal del desplazamiento en muchas partes del Pacífico (Escobar, 2010).

Por su parte, Castellanos $(2015 ; 2018)$ ha estudiado los casos de las regiones del Soconusco y Marqués de Comillas (Chiapas, México), y encontró que la expansión de la palma aceitera no ha detonado acciones violentas, sino más bien

\footnotetext{
2 Robbins (2004), Escobar (2010), Alimonda (2017) y Leff (2017).

3 Hemos optado por este nombre, que identifica a los productores de pequeña escala, ya que en los diversos diálogos durante el trabajo de campo lo que más resaltó es que quienes cultivan palma se identificaban en mayor medida como campesinos y como palmeros. Otra tendencia fuerte fue el identificarse como productores (de palma).
} 
conduce a diversas negociaciones entre el Estado mexicano y la población rural, entre quienes destacan los ejidatarios involucrados en la producción agrícola. Su análisis se centra en esclarecer las complejas articulaciones entre el Estado y las poblaciones locales ante las emergentes intervenciones productivas y ambientales. No pretendemos negar la existencia en la región de Palenque de «conflictos de distribución ecológica» (Martínez-Alier, 1997) en los que la gente se moviliza en contra de los aspectos destructivos de la globalización encarnada en el auge de la agroindustria aceitera, sin embargo consideramos que en esta primera aproximación a la zona de estudio era estratégico no centrarnos en los conflictos socioambientales (por lo delicado del tema y su complejidad). Nos pareció pertinente primero indagar cómo se está configurando la territorialidad del cultivo de palma, lo cual no nos impidió partir del reconocimiento de que las tensiones sociales que han marcado la historia reciente de esta región tienen raíces de hace años (nos basamos en estudios previos para reconstruir una narración histórica), ni tampoco entender que hay múltiples actividades en la zona $y$, menos, ver los conflictos vinculados al cultivo.

Si consideramos lo anterior, el abordaje es de carácter territorial en tanto que para este estudio de caso buscamos articular aspectos agrícolas, agrarios, sociales y ecológicos. Dentro de este acercamiento territorial también retomamos las cuestiones económicas y políticas, resaltadas en estudios sobre otros cultivos (Friedmann, 1982; McMichael, 1994; Mier y Terán Giménez Cacho, 2016; Oliveira y Hecht, 2016); y que también han sido determinantes en el auge de la palma aceitera, ya que es un cultivo promovido por la agroindustria en una fina articulación con el poder que emana del Estado en forma de políticas públicas. Pero, para entender cómo se está dando la territorialización, nos posicionamos dentro de los estudios que buscan explicar el auge de la palma aceitera mediante la identificación de las implicaciones en la vida del campesinado (Castellanos, 2018:3).

A partir de Haesbaert $(2011,2013)$ abordamos el presente trabajo desde la óptica de la territorialización y desterritorialización; herramienta analítica que articula en gran medida la narración con perspectiva histórica que se desglosa en esta investigación. De acuerdo con el autor, los procesos de territorialización suceden de manera integral, es decir, funcional y simbólicamente; él clasifica lo funcional y lo simbólico como vertientes complementarias en la construcción de territorios. Lo simbólico engloba la construcción de imaginarios y representaciones sobre el territorio, es la carga cultural sobre el espacio apropiado; mientras 
que lo funcional es la carga material, la delimitación física del espacio apropiado y la determinación de las funciones que este debe desempeñar (Haesbaert, 2013).

Asimismo, partimos de que territorialización, desterritorialización y territorialidad derivan del concepto de territorio (Ramírez y López, 2015:151). Nuestra postura conceptual acerca de este radica en que es construido funcional y simbólicamente por diversas relaciones socioambientales que cuentan con su propio contexto histórico. Alejándonos así de las dicotomías en torno al territorio en cuanto a lo social y lo natural, tiempo y espacio, lo móvil y lo inmóvil (Haesbaert, 2013). En este sentido, es importante señalar que en cuanto a la relación entre territorio y poder, Haesbaert (2013:26) indica que el primero debe ser entendido como producto «de las relaciones de poder construidas en y con el espacio, considerando el espacio como un constituyente, y no como algo que se pueda separar de las relaciones sociales».

El carácter interdisciplinario de la ecología política permite asumir el reto del objetivo de esta investigación, la cual propone ver a la agroindustria aceitera como una red física, simbólica e institucional que permea la realidad local, y que se expresa visiblemente en la expansión y consolidación de monocultivos de pequeña y mediana escala, pero que, además, constituye la emergencia de dinámicas socioterritoriales con cargas de poder diferenciadas entre actores locales, extraterritoriales, de mercado y del Estado.

\section{Metodología}

Nuestra investigación es cualitativa con énfasis en la aplicación de entrevistas semiestructuradas, así como algunas técnicas etnográficas como las pláticas informales, las observaciones en campo y el registro en el diario de campo.

El análisis fue a partir de información del trabajo de campo realizado entre marzo y junio de 2018 y, como complemento, se incluye información proveniente de diálogos y observaciones de una etapa exploratoria en 2017. El registro de trabajo de campo se compone de 21 entrevistas, siete caminatas guiadas en plantaciones de palma aceitera, transcripciones de varias pláticas informales, actividades de observación y notas de tres asambleas regionales de palmeros a las que asistimos. La información derivada de estas fuentes se vinculó con literatura secundaria para hacer más robusto el análisis de los hallazgos en campo 
(Stake, 1998:98). Las asambleas regionales se celebraron el 1 de abril, el 6 y el 20 de mayo de 2018, todas en la ciudad de Palenque. ${ }^{4}$ Las dos primeras fueron en fechas ordinarias, mientras que la última fue extraordinaria. En esta asamblea hubo elección y oficialización de los representantes regionales, quienes tomaron protesta en presencia del presidente del Consejo Nacional de Productores de Palma de Aceite (quien a su vez es el presidente del Consejo Estatal de productores del estado de Chiapas), de algunos delegados del gobierno municipal de Palenque y de la Secretaría de Agricultura, Ganadería, Desarrollo Rural, Pesca y Alimentación (SAGARPA). ${ }^{5}$

En su mayoría, las entrevistas se realizaron en localidades rurales del municipio de Palenque (además de un par en la ciudad). En dos ocasiones se hicieron actividades de investigación fuera de este municipio para llevar a cabo tres entrevistas en San Jerónimo Tulijá, municipio vecino de Chilón; y una caminata guiada en un «rancho ${ }^{6}$ ubicado en el municipio de Playas de Catazajá, también aledaño.

Este estudio de caso $^{7}$ se concentra en la región norte de Chiapas, específicamente en Palenque. A continuación presentamos el contexto que permite abordarlo como un espacio apto para estudiar la territorialización de la agroindustria de la palma, para después continuar con la identificación de cómo este cultivo se territorializó en esta región.

\section{La palma de aceite en el sureste mexicano}

En México, la producción de aceite de palma ha aumentado paulatinamente para cubrir parte de la demanda de aceite vegetal de las industrias asentadas en el país (Trejo, 2018). Sin embargo, el déficit de la producción de esta oleaginosa

${ }^{4}$ De manera habitual se procura que todas las asambleas se realicen cada primer domingo de mes.

5 Ahora con el nombre SADER (Secretaría de Agricultura y Desarrollo Rural).

${ }^{6}$ Localmente quienes trabajan en las plantaciones comerciales que pertenecen a los corporativos agroindustriales nombran estos espacios como 'ranchos'.

7 Un estudio de caso es, desde la perspectiva de Stake (1998:11) «el estudio de la particularidad y de la complejidad de un caso singular». En el mismo sentido, Neiman y Quaranta (2006:217) nos ayudan a afianzar la selección de esta metodología al indicar que «la diversidad de significados otorgados y posiciones abarcadas por el 'estudio de caso' cubre un amplio espectro de campos y enfoques, que puede comprender desde análisis teóricos y de carácter macro-históricos hasta investigaciones empíricas sociológicas e incluso etnográficas». Así asentamos la sincronía entre investigaciones cualitativas, la ecología política y el recurso analítico de los estudios de caso sobre territorialidad. 
ha tenido que complementarse con importaciones significativas, en especial de países de Latinoamérica (Trejo, 2018). Esta situación parece indicar que hay suficiente interés por las empresas usuarias de este insumo para que la producción de palma siga en aumento, como se demuestra en las cuentas nacionales sobre la superficie sembrada y cosechada de este cultivo. Visto en cifras, para 1999 la superficie cultivada no había superado las 10 mil hectáreas (desde entonces Chiapas ya figuraba como la entidad federativa con mayor superficie cultivada). Entre el 2000 y 2004 se registró un aumento superior al 100 \% (partiendo de unas 16 mil ha hasta subir a más de 36 mil ha). Nueve años después, en 2013, las hectáreas sembradas se duplicaron a más de 74 mil (Linares et al., 2018; SIAP, 2019). En 2018 las hectáreas sembradas superaron las 101 mil; de las cuales Chiapas tiene $45 \%$, mientras que Campeche y Tabasco tienen $27 \%$ y $21 \%$, respectivamente, y Veracruz tiene 7 \% (SIAP, 2019). Es decir, 100 \% de la expansión de la palma de aceite está concentrada en el sureste mexicano. En este aumento de un par de décadas, Chiapas ha sido el mayor contribuidor, aunque en los recientes años ha disminuido la proporción que aporta a nivel nacional (Linares et al., 2018).

Durante el sexenio de Juan Sabines (2006-2012), el gobierno de Chiapas impulsó en gran medida la producción de biocombustibles ${ }^{8}$ con énfasis en el cultivo del piñón (Jatropha Curcas), un proyecto que con el paso de los años no tuvo éxito. Mientras tanto, la palma aceitera se consideró supletoria para tal uso (Santacruz de León y Palacio, 2018). Dicho impulso gubernamental contribuyó a que la superficie sembrada de piñón aumentara aproximadamente 10000 ha aunque, paradójicamente, la palma aceitera aumentó unas 30000 ha entre 2007 y 2012 (Castellanos y Jansen, 2017). Entonces, ¿por qué elegir al municipio de Palenque para buscar entender la territorialización de la palma? En la zona de estudio vemos que entre los años 1950 a 2000 los principales fenómenos que han moldeado los territorios rurales (y urbanos) son la migración interregional y la nacional, la transculturación de pueblos indígenas, ${ }^{9}$ y el escalamiento de la llama-

8 El mercado internacional de biocombustibles es un factor importante en la fluctuación del precio del aceite de palma. Los altos precios del aceite han llegado a generar que «en diversas áreas del mundo se impulse la reconversión de tierras al cultivo de la palma, aun aquellas que son ecológicamente sensibles» (Santacruz de León y Palacio, 2018: 84).

9 La transculturación en este caso sucede por los contactos y relaciones entre distintos ámbitos culturales, en general entre campesinos mestizos, campesinos indígenas y agentes del sector agroindustrial. Los cambios debieron darse en múltiples dimensiones que implican relaciones de poder y de dominio (Pérez-Brignoli, 2017). 
da revolución verde. En este último se enmarca el paquete tecnológico ofrecido por la agroindustria de la palma y su consecuente modelo de producción. ${ }^{10}$

En la revisión del estado de la cuestión sobre la palma aceitera en México, identificamos que la región de Palenque ha sido estudiada con menos intensidad. En la selección de la zona también consideramos factores sociales, en específico la mayor concentración étnica (ch'oles y tseltales), lo cual nos permite estudiar si la territorialización de la palma está generando reconfiguraciones de las relaciones de producción agrícola de esta población. Esto refuerza el interés de enfocarnos en los campesinos-palmeros como sujetos que están viviendo la territorialidad de la agroindustria de palma, aunque para ampliar la visión también tomamos en cuenta otros sujetos que han conocido de cerca algunas particularidades de esta expansión.

La expansión de la palma se ha dado en zonas específicas de Chiapas, y si bien cada una tiene su historia agraria, la de Palenque es distinguible a la de las regiones Costa-Soconusco y Marqués de Comillas. Entre las regiones de Palenque y el Soconusco existe similitud histórica en cuanto al auge de la cultura finquera del siglo XIX; pero, si nos detenemos a hacer lecturas más precisas, podremos notar matices entre cada región (Washbrook, 2018). De todo el territorio mexicano, fue la región del Soconusco en donde se comenzó a cultivar la palma aceitera (Gobierno Constitucional del estado de Chiapas, 1991); una proporción importante de los campesinos que apostaron por la producción de la palma habían obtenido sus pequeñas parcelas por el (tardío) reparto agrario que consagró tierras ejidales a mediados del siglo XX. En contraste, la región de Marqués de Comillas fue 'colonizada' entre las décadas de 1960 y 1970, cuando grupos poblacionales (originarios de otras regiones de Chiapas y de diversos estados de la República mexicana) fundaron ejidos que abarcaron la región más oriental de Chiapas en los límites con Guatemala (Cano, 2014). En Marqués de Comillas la palma aceitera comenzó a expandirse desde el 2005 y fue impulsada, localmente, por organizaciones rurales que la habían identificado como una alternativa económica para la gente de esta región (Castellanos, 2015). Algo característico de la región del Soconusco son los casos de propiedad social de algunas plantas extractoras, que en el caso de Palenque es una aspiración expresada tanto en las asambleas de la organización regional de

10 Esta delimitación va de la mano con los temas resaltados por varios interlocutores durante el trabajo de campo de esta investigación. 
productores de palma, como en entrevistas a productores que afirmaron ser parte de la organización regional.

\section{Antes de la palma: reconfiguraciones agrícolas y agrarias de las re- cientes décadas}

A partir de 1996 la palma aceitera comenzó a ser parte de los paisajes agrícolas de la región de Palenque, en específico en la zona del Valle de Tulijá, (Linares, et. al, 2018). A lo largo de los últimos dos siglos, distintas actividades humanas han impactado el territorio: desde la extracción de maderas preciosas y el auge de fincas privadas, hasta la más reciente ganaderización de la selva entre las décadas de 1950 a 1980 (De Vos, 1988; Villafuerte et al., 1997; Fenner, 2015; Washbrook, 2018). En este sentido, la expansión de la palma aceitera implica una serie de actividades que repercuten en intervenciones territoriales, esto con algunas particularidades que resaltaremos más adelante.

Décadas antes de la llegada de la palma aceitera a Palenque, entre 1950 y 1980, se dio un proceso de ganaderización que impactó en diversas y amplias proporciones de selva alta y mediana (Villafuerte et al., 1997). Don Chambuchín, -como es conocido localmente el cronista municipal de Palenque- cuenta que la ganadería incidió en la deforestación de las serranías, esto bajo la lógica de que primero ocurrió una etapa fuerte de ganaderización en las planicies:

¡Suban las vacas al cerro! [aludiendo esta frase a agentes de gobierno] y las vacas todo lo que comen allí [arriba] lo queman en la bajada, y lo que comieron abajo lo queman en la subida. Entonces no resulta en negocio para el ganadero, pero ya destruyeron el cerro (entrevista, 22/06/2018, ciudad de Palenque).

Esta ola de ganaderización se dio cuando llegaron proyectos impulsados por el Estado mexicano. El diácono de la región, ${ }^{11}$ habitante de la zona del Valle de Chancalá, también toca el tema de la ganadería y manifiesta que él observó las consecuencias de esta actividad: «desaparecieron muchas especies de árboles,

${ }^{11}$ Por encargo de la iglesia católica este diácono realiza sus labores en la región que incluye los municipios de Tila, Yajalón y Tumbalá, Palenque, La Libertad, Playas de Catazajá, Salto de Agua y Chilón, así como el norte del municipio de Ocosingo y los municipios del sur y del este de Tabasco colindantes con Chiapas. 
manantiales y se secaron varios arroyos» (entrevista, 8/05/2018). En cuanto a las actividades agrícolas de la región, el diácono indicó que el uso de agroquímicos generó cambios significativos en las formas de realizar las jornadas laborales en el campo, a la vez que rememora cambios importantes desde que él tiene vida. A saber, primero llegó una bomba que se colgaba de un solo hombro, luego una para colgarse en dos hombros (la «mochila», como se nombra localmente), después llegó la moto que en poco rato rociaba toda una hectárea. Todo esto acompañado de promesas de mejoras, "y ahora se viven las consecuencias en la salud», arguye (entrevista, 8/05/2018).

Además de la adopción de la ganadería intensiva, por Palenque han transitado diversos cultivos agrícolas; según los entrevistados, algunos de los más importantes han sido el arroz, el café, el hule y el chile. Si tomamos el ejemplo del ejido La Cascada (Palenque) hay gente que afirma que hace un par de décadas el cultivo comercial predominante era el chile, lo cual contrasta con años recientes en los que la palma de aceite ha tomado el papel central:

[la gente] sembraba lo que era frijol, maíz y chile, pero lo que pasa [es que] el precio del picante está muy bajo hasta ahorita. Digamos, tiene 17 años que no ha subido, apenas va a \$1.50, creo, el kilo del chile verde. Está muy barato y lleva mucho pesticida y todo eso, y no sale nada [...]. Pero la mayor parte de la gente dejó de sembrar todo eso (entrevista, 6/06/2018).

En el ámbito agrario, en Palenque se fundaron diversos ejidos a mediados del siglo XX a partir de la dotación y ampliación de tierras ejidales. Sumado a lo anterior, en la segunda mitad de la década de 1990, y en el contexto del levantamiento armado del Ejército Zapatista de Liberación Nacional (EZLN), se fundaron algunos ejidos tanto en las planicies como en la serranía. Así, desde 1996 diferentes agrupaciones campesinas ocuparon tierras de uso finquero, donde muchas veces predominaba la actividad ganadera. Esta reciente etapa de reconfiguraciones agrarias y poblacionales también implicó la concentración de grupos de población con características étnicas distintas, es decir se dio la confluencia de pequeñas migraciones al interior de la región. Una tendencia a destacar es la movilización de grupos ch'oles y tseltales que se trasladaron de zonas altas a valles como el de Chancalá y el Tulijá, así como a planicies de Palenque. Mientras que varios grupos de campesinos mestizos también forma- 
ron parte de estas reconfiguraciones poblacionales, conformando los nuevos ejidos en las planicies. ${ }^{12}$

En estos ejidos, fundados en dos periodos distintos (1930-1980 y 1996-2000), es donde actualmente se localiza una proporción considerable de las plantaciones comerciales de palma aceitera. Fundados dentro del periodo 1930-1980, los ejidos del municipio de Palenque que abarca el trabajo de campo de esta investigación, son: Chancalá, Emiliano Zapata, La Cascada y Saturnino Ríos. Mientras que los ejidos Miguel Hidalgo II, San Mateo II, Filadelfia, Victoria Campesina II y Chancalá Zapote, fueron conformados en el contexto de las reconfiguraciones territoriales en la etapa de posconflicto armado de 1994. También se hicieron visitas en otros municipios, a los ejidos San Jerónimo Tulijá (Chilón) y Suclumpá (Salto de Agua), constituidos a mediados del siglo XX, y al ejido Río Tulijá (Salto de Agua), creado en las reconfiguraciones territoriales después de 1994. En cuanto al municipio de Palenque, muchos de los ejidos fundados en tiempos de posconflicto armado se crearon en 1996; esto a partir de diversas negociaciones entre campesinos y propietarios privados que mantenían en actividad diversas fincas. Aquí cabe mencionar que el Estado mexicano fue mediador clave en estas negociaciones. ${ }^{13}$

En síntesis, las reconfiguraciones agrícolas, agrarias y sociales en las recientes décadas son parte importante para entender por qué la palma de aceite encontró cabida en los territorios agrícolas de Palenque. En otras palabras, ya existían procesos que predefinieron la permeabilidad de este territorio hacia la actividad agroindustrial en torno a la palma aceitera.

\section{El papel del Estado mexicano en la llegada de la palma a Palenque}

Como ya habíamos adelantado, fue en una zona específica del Valle de Tulijá (municipio de Salto de Agua) donde iniciaron las primeras plantaciones de palma aceitera, y donde el Estado mexicano impulsó la instalación de viveros que abastecerían de plántulas a toda la región de Palenque. Antes del 2000, la palma aceitera ya se había establecido en Salto de Agua, Palenque, Chilón y Playas de Catazajá. Previamente, este cultivo solo tenía presencia en la región

12 En cuanto a las reconfiguraciones poblacionales, sobresale lo sucedido en las décadas de 1960 y 1970 cuando se asentaron diversos grupos en el Valle de Chancalá, en su mayoría provenientes de regiones del centro del país.

13 Sobre las invasiones y compras de tierras en Palenque véase Villafuerte et al. (2002). 
del Soconusco. ${ }^{14}$ Dentro de los relatos de los campesinos-palmeros de Palenque hay una generalidad que señala el papel de los agentes del Estado mexicano como impulsores de la llegada de la palma aceitera a la región. De acuerdo a entrevistas y pláticas, la mayoría de las plantaciones se establecieron entre 1998 y 1999 para el caso del municipio de Palenque. Uno de los productores del ejido Saturnino Ríos, de las planicies de Palenque cercanas a Tabasco, describe algunos detalles acerca de los inicios de la palma en su localidad:

Vinieron a hacer como una encuesta aquí, a ver quién estaba interesado en esa plantación que no la conocíamos, en el 98-99 [...]. El gobierno nos regaló la plantación [las plántulas] y nos pagó quizás como cuatro años de mantenimiento para fertilización y limpieza hasta que llegara la comercialización, porque aquí no había nada de plantas extractoras ni nada [...]. No lo habíamos visto nosotros en realidad cómo se manejaba esto, solamente por pláticas teníamos confianza de que se iba a hacer negocio (entrevista, 21/06/2018).

Una de las vías de entrada de la palma de aceite a las localidades rurales de Palenque fueron los proyectos productivos ofrecidos por distintas dependencias gubernamentales. Esta tendencia es evidente dado que se presentó a la palma como una de las pocas opciones productivas para las comunidades fundadas desde 1996 (dentro de las reconfiguraciones de tenencia de tierras ya mencionadas). El relato que más pistas nos da es el de don Paco, quien indica que agentes de COPLANTA (Centro de Investigación y Desarrollo de Plantaciones) llegaron a ofrecer asesoría y plántulas de manera gratuita en uno de los ejidos que se encontraba en proceso de legalización:

Entonces ya empezó "miren, las plantas regaladas, no paguen nada; el acarreo, el camión, no van a pagar nada si ustedes le entran" [él atribuye esta frase a agentes de COPLANTA]. Yo me acuerdo que nosotros le entramos y en ese momento venían camiones llenos de plantitas, y lo tiramos allí en la zona escolar. "¡Ay 'ta! ¡Y siémbrenlo!". Bueno, vinieron ya los técnicos [...] a enseñar cómo se va a trabajar, cómo se va a balizar y cómo se va a sembrar. Le entramos. Empezamos a limpiar (entrevista, 27/03/2018, ejido Filadelfia).

\footnotetext{
14 A principios de la década de 1990 los planes y estudios para la expansión de palma aceitera se enfocaban en la Costa y el Soconusco (Ramírez, 1991) y fue hasta el 2005 que se introdujo este cultivo en la región de Marqués de Comillas (Castellanos, 2015). Mientras que las plantaciones de palma aceitera en la región de Palenque comenzaron a establecerse en 1996, del lado sur, en el Valle del Tulijá (Linares et al., 2018).
} 
El papel del Estado mexicano fue el común denominador en la llegada de la palma aceitera a Palenque, manifestado principalmente en acciones de instancias a nivel municipal y estatal. La producción de materia prima era el preámbulo que en 2004 aprovecharían las plantas extractoras del sector privado que llegaron bajo gestiones gubernamentales: Palma Tica y Agroipsa.

En síntesis, la palma aceitera se promocionó en estas comunidades con un modus operandi característico para la zona norte de Chiapas: exponer las ventajas productivas y económicas de la palma, ofrecer paquetes tecnológicos y créditos a fondo perdido para los primeros años de cultivo de las plantaciones y otorgar las plántulas gratis. Los agentes empresariales llegaron a la par de los agentes gubernamentales bajo la figura de asesores técnicos y proveedores de paquetes tecnológicos.

\section{El papel de las plantas extractoras y los centros de acopio}

El fruto de la palma aceitera tiene como fin servir de materia prima al siguiente eslabón dentro de la cadena de producción. Después del inicio de las plantaciones comerciales de palma aceitera, en la región de Palenque se debía construir una planta extractora de aceite, que sería la encargada de comprar y transformar la cosecha de aquellas plantaciones. El relato de don Paco nos permite entender que los campesinos-palmeros vivieron un par de años de incertidumbre al no saber cómo y a quién vender los frutos que pronto cosecharían, la mediación por parte del gobierno mexicano era menester para solucionar este dilema:

A los tres años empezó a tirar la producción. Luego se nos presenta nuevamente el problema: ¡¿dónde vamos a vender?! [...]. Entonces la Secretaría del Campo dijo: "No, pues el gobierno no les va a dar nada, simplemente el gobierno les va a abrir un espacio para gentes que tienen dinero, que sepan el negocio y que pongan su planta extractora, y que ellos se encarguen de comprar" [...]. Hubo como dos-tres años de puras organizaciones, jreuniones, reuniones! Hasta que vino en una ocasión una empresa costariquense y luego vino también una empresa mexicana que se llama Agroipsa, que tenía su matriz en Guadalajara (entrevista, 27/03/2018, ejido Filadelfia).

Algunas cosechas se perdieron antes que las plantas extractoras comenzaran a funcionar. En el ejido Saturnino Ríos no solo fue eso, sino que muchos produc- 
tores desistieron de seguir con la producción, aunque también otros aguantaron hasta la llegada de la extractora de Palma Tica. Don Hernán cuenta que:

mucha gente se desanimó porque no miraban quién iba a comprar el producto y varios aguantamos la espera hasta que llegó, en ese entonces se llamaba Palma Tica ahora creo que es Palmosur, y ya empezó a comprarla y no se ha parado la compra de eso hasta el día de hoy (entrevista, 21/06/2018).

Otros ejidos también abandonaron la actividad palmera, dos tendencias claras respecto a esta decisión son la pérdida de cosechas y la ocurrencia de plagas (en especial de roedores).

Al principio (1996-2003) solo existían las plantaciones comerciales de los productores locales, mientras que las industrias iniciaron sus actividades de extracción de aceite hasta 2004 (Agroipsa y Palma Tica). La llegada de las extractoras estimuló la creación de centros de acopio. Trece años después, en 2017, entró en escena una tercera planta extractora en la región, de la empresa Uumbal, ubicada en el extremo noreste que colinda con el municipio de Emiliano Zapata, Tabasco. Las plantaciones, las acopiadoras y las extractoras son los espacios donde la materialización de la agroindustria aceitera es más clara.

Muchos de los centros de acopio tienen acuerdos de comercio exclusivo con las tres empresas que tienen influencia en la región: Palmosur, Oleopalma y Uumbal. El centro de acopio de La Cascada tiene acuerdos directos con Uumbal desde el 2017. Casos similares, donde el centro de acopio surte de fruta a una empresa en particular, son los centros ubicados en Filadelfia y en Saturnino Ríos. Por lo general, los productores de la región comercian su fruta sin estrictos acuerdos comerciales con las empresas extractoras, así lo afirma el encargado del centro de acopio del ejido La Cascada: «aquí es libre si quieres vender la fruta, lo que sí es que tienes que registrarte porque si no, no te reciben la fruta» (entrevista, 6/06/2018). Para él, en 2017, fue relativamente fácil dejar su relación comercial con Agroipsa (ahora Oleopalma) para comerciar la fruta que acopia con Uumbal, y no tuvo que rendir cuentas con Agroipsa por hacer este cambio.

La relación entre empresas aceiteras y productores de palma va más allá del comercio mediado por los centros de acopio; a continuación veremos que un factor clave para la relación entre estos dos grupos es el precio de la fruta, además de otros factores logísticos que destacamos. 


\section{Organización regional y tensiones entre sector empresarial y productores} de palma

Dentro de las dinámicas sociales en torno a la palma aceitera están los espacios organizativos de los productores. Algunos solo se organizan para vender su fruta y normalmente se autodenominan productores independientes, mientras que otros se reúnen a nivel regional con objetivos más ambiciosos, tales como tener su propio vivero de plántulas de palma o constituir una planta extractora con carácter social (donde los socios mayoritarios sean los mismos productores).

La sociedad regional de productores de palma de aceite congrega prácticamente a todos los municipios de la región (Playas de Catazajá, Palenque, La Libertad y Salto de Agua). ${ }^{15}$ Esta sociedad aglutina a cerca de 15 Sociedades de Producción Rural (SPR), y se reúnen en asambleas casi cada mes. Hasta principios de 2018 gran parte de lo que se conoce como Valle de Tulijá contaba con sus propias SPR pero estaban distanciadas políticamente de la sociedad regional.

De acuerdo con el representante de la región, estas sociedades conformadas dentro de los ejidos se han unido como sociedad regional con el fin de concretar algunos proyectos y también para "defenderse de los empresarios» (entrevista, 8/04/2018). En sintonía con esta postura, un productor de San Mateo II afirmó que los productores de pequeña escala están en clara desventaja competitiva con la producción de las plantaciones que administran las empresas aceiteras de la región; además, este productor indica que una razón clave de la existencia de la sociedad regional es lograr una demanda social fuerte para que los precios de la fruta sean justos (entrevista, 27/04/2018).

Quienes están organizados en la sociedad regional de productores tienen metas que van más allá del aumento en el número de hectáreas sembradas y del mejoramiento de las condiciones productivas. Varios de los campesinos-palmeros que conforman la sociedad regional, plantean que los beneficios de cultivar palma aceitera podrán mejorar si hay una organización sólida de productores de la región. Y es el aspecto organizativo lo que puede posicionarlos mejor frente a dificultades competitivas con las plantaciones empresariales y al poco cobijo por parte de los aparatos del Estado mexicano.

\footnotetext{
15 No se encontró evidencia de que algún campesino-palmero del municipio de Chilón formara parte de dicha sociedad regional.
} 
Varios productores, entre los que destacan nuevamente quienes están integrados en la organización regional, aseguran que actualmente (2018) han recibido poca asistencia por parte del Estado mexicano, a diferencia de los primeros años de implementación de la palma, cuando el apoyo era muy claro en términos materiales y financieros. Y el hecho de que el gobierno ya no dé las plántulas gratis ha resultado ser un problema, sobre todo para la proporción de los productores de pequeña escala que buscan aumentar sus hectáreas sembradas.

Por lo tanto, las aspiraciones de los productores organizados en la sociedad regional de Palenque son, a corto plazo: tener su propio vivero, consolidar su registro como sociedad mercantil a nivel federal, abastecerse de mejor manera de paquetes tecnológicos (de fertilizantes certificados, por ejemplo) y solicitar al gobierno la generación de proyectos para los productores de pequeña escala; a mediano plazo, tener una planta extractora propia y, a largo plazo, contar con una planta refinadora de aceite.

Los campesinos-palmeros regulan su participación en la cadena de producción del aceite de palma con base en su interacción con los empresarios, los agentes del Estado mexicano y con otros tipos de productores en la región (los ganaderos, por ejemplo). En cuanto a las problemáticas surgidas entre el sector empresarial y los productores de pequeña escala, en el 2016 hubo una manifestación debido a los precios que pagaban las empresas a los productores y a las condiciones que tenían que soportar estos últimos en espera de que les recibieran la fruta:

La primera vez que intentamos organizarnos no funcionó. Luego, a raíz de que hicimos plantones y todo por el maltrato que daban aquí en Agroipsa, por el mal pago y todo eso, nos volvimos a reorganizar, que es en donde estoy ahorita, que va para dos años en agosto que volvimos a reiniciar, pero ahí sí ya es una organización grande porque ya éramos más de 1000 palmeros [que] nos juntamos, no solo de la zona [del municipio] de Palenque sino lo que es La Libertad, lo que es Salto de Agua, toda esa zona por allá y pues fuimos un grupo grande (entrevista, 26/04/2018, ejido San Mateo II).

Este relato ejemplifica uno de los momentos críticos en la relación entre empresarios y productores de pequeña escala: las manifestaciones en contra de las empresas extractoras en 2016 (Agroipsa y Palma Tica), ya que estas compraban la fruta en pequeñas cantidades mientras que los productores requerían vender 
mucha porque era temporada alta de cosecha. Las empresas no necesitaban aumentar los volúmenes de compra, ya que sus plantaciones aportaban buena parte de lo necesario para su capacidad de extracción de aceite. El hartazgo de los campesinos-palmeros no fue repentino ya que, además, se sumó el sentir colectivo de que sus frutas no eran pagadas a buen precio.

Para los productores de pequeña escala el ámbito organizativo fue determinante para encaminar las soluciones financieras y logísticas desde el inicio de sus plantaciones, sin embargo, se vieron envueltos en dificultades relacionadas con el oportunismo de líderes y con casos de corrupción. Quienes hasta el presente se organizan en la sociedad regional de productores también viven dificultades, muchas ocasionadas por acciones empresariales (como la variación de precios o el cierre temporal de compra de frutas en época de alta producción) que son permitidas y consentidas por el Estado mexicano, según afirmaron en varias entrevistas y pláticas. En este sentido, cabe destacar que el Estado, más allá de ser permisible, es la figura política que ha construido la idea de desarroIlo basado en el libre comercio; de acuerdo con esto, el Estado no interviene específicamente en el comercio local de la palma, pero sí fomenta la estructura comercial donde el aceite vegetal es una mercancía importante para el modelo de desarrollo predominante.

Organizarse, asociarse, reunirse, capacitarse, todo esto sucede en los espacios sociales dinamizados a partir de la expansión de la palma. Son parte de las formas en que la palma va adquiriendo significados específicos en la vida de quienes producen los frutos y de quienes están relacionados de alguna manera a toda la cadena de producción. Las relaciones directas entre la palma y la población local son más palpables en el ámbito agrícola. En el siguiente apartado ampliaremos acerca de cómo los productores de pequeña escala describen su participación en relación a su coexistencia con la producción de aceite de palma en su conjunto.

\section{La actividad palmera en el campesinado}

Existen distintas valoraciones sobre las plantaciones comerciales de palma aceitera en Palenque. Si nos enfocamos en quienes la cultivan podemos identificar también los matices: aspectos productivos, ambientales, sociales, y, por supuesto, los económicos. Dichos matices son los que podemos destacar como tendencias a hibridismos en relación a las territorialidades locales que han sido 
construidas a partir de una cultura campesina o ganadera sobre territorios donde destaca el avance de un modelo agrícola basado en una lógica agroindustrial. De los relatos de los campesinos-palmeros emergieron temas en torno a las necesidades familiares y comunitarias que deben atenderse acorde a las posibilidades. Es así como uno de los líderes de antaño, don Paco, cuenta cómo trató de cultivar maíz, sin mucho éxito, a la vez que intentaba obtener un ingreso económico extra al pepenar en el basurero localizado a medio kilómetro de su casa. La palma llegó cuando don Paco necesitaba con urgencia una mejora económica y «le ha ido bien». Él dice:

nos alcanza para una familia chica, por decir: yo y mi familia (mi esposa y mis dos hijas) [...]. Estamos viendo de que [sic] es algo redituable el trabajo, no digamos para hacernos ricos, pero para justamente mantener la familia, los hijos. Yo me acuerdo que mis niñas pues aquí nacieron, les di el estudio, y una muchacha que tengo pues gracias a Dios está terminando su carrera, y ella [la hija menor, que estaba presente durante la entrevista] va a terminar la prepa también (entrevista, 27/03/2018, ejido Filadelfia).

Don Paco, originario de la serranía de Palenque, se mudó a la planicie para fundar el ejido Filadelfia junto con otras personas provenientes de diversas zonas del municipio. Él asevera que formó parte de los líderes que negociaron con el gobierno federal y los propietarios de tierras a quienes se les expropiaron ranchos en diversas zonas de Palenque. Además, fue líder de la SPR local a principios de la década del 2000. En la actualidad se autodenomina como productor independiente.

Otro ejemplo es el descrito por el líder regional, él asegura que tanto en lo económico como en lo social la palma aportó mucho a las economías familiares del ejido Filadelfia (donde él ha vivido más de una década) y, además, esto repercutió en que los pobladores locales ya no migraran para trabajar en otras zonas de Chiapas y de México (entrevista, 8/04/2018).

Ahora bien, ha habido algunas dificultades de tipo productivo para los campesinos-palmeros, la principal tuvo que ver con la emergencia de plagas. Las ratas invadieron varias hectáreas entre las plantaciones de la región. Muchas de las plagas fueron controladas, pero una proporción considerable afectó a tal grado que fueron una razón importante para que algunos productores desistieran en la apuesta por la palma, esto concluyen algunos de los productores que sí aguantaron el combate contra las plagas. Cronológicamente, los relatos que hacen referencia a quienes desistieron se ubican entre 1996 y 2004, esto tanto 
por las plagas como por la pérdida de las primeras cosechas antes de la llegada de las plantas extractoras.

Los matices de las trayectorias individuales sobre la expansión de la palma aceitera apuntan, por un lado, a que la palma es considerada por algunos campesinos-palmeros como un cultivo con una presencia positiva en comparación con otras actividades, como la ganadería; para otros, es un mal necesario para sobrevivir; y, por otro lado, hay opiniones que contrastan con las anteriores ya que, quienes no siembran palma, señalan otras problemáticas de tipo ambiental y ven que el balance es más bien negativo.

A pesar de que la producción de palma aceitera es una de las principales actividades agropecuarias en Palenque, ${ }^{16}$ no ha sido un motor de enriquecimiento económico para todas las familias campesinas involucradas, no obstante sí logra satisfacer algunas necesidades básicas, dentro de las cuales los productores identifican la compra de vehículos pequeños, el pago de gastos escolares, la compra de alimentos de la canasta básica, entre otras. En otras palabras, la percepción expresada es que la producción de palma sí ayuda económicamente al campesinado de Palenque, pero sin que esto signifique «riqueza», esta es una tendencia importante dentro de las percepciones locales tanto entre quienes la cultivan como entre quienes no. Mientras más hectáreas sembradas se tengan, mayores son las posibilidades de aumentar las ganancias económicas, situación que para muchos implica tener al menos 10 hectáreas en cosecha.

\section{La producción sustentable de la palma, fundamentos y contradicciones}

La controversia ambiental surgida por la presencia de monocultivos de palma aceitera y su avance en la región sigue latente. El sector empresarial y parte de los productores de fruta mantienen discursos que consideran a la palma aceitera como beneficiosa para el ambiente. Testimonio distinto del de algunos otros productores del fruto y de algunas personas de la región que reconocen los impactos ambientales de las plantaciones comerciales y de la infraestructura de las extractoras de aceite. Respecto a afectaciones ocasionadas por esta última, el caso emblemático fue la contaminación a casas-habitación de un ejido colindan-

16 En tanto producción agrícola, la palma es el segundo cultivo de importancia en Palenque, solo detrás del cultivo de maíz grano blanco, mientras que el frijol es el tercer cultivo de importancia en este municipio (SIAP, 2019). 
te a la extractora de Palmosur. Las lagunas de oxidación se desbordaron en época de lluvias, lo que condujo a las aguas residuales hasta las casas y traspatios de los habitantes (entrevista, 19/06/2018, ejido Victoria Campesina II). Las aguas residuales llegaron a contaminar el río Michol (tributario del río Tulijá), lo que al parecer causó la muerte de peces y demás fauna acuática en la zona (entrevista, 21/06/2018, ejido Saturnino Ríos).

En general, la visión de quienes indican algunas ventajas ambientales de la palma aceitera está directamente relacionada con la noción de «producción sustentable de la palma de aceite». Esta implica cuidar ciertas condiciones en las plantaciones y en el procesamiento para la obtención del aceite y está ligada a satisfacer políticas comerciales de responsabilidad social y ambiental (entrevista, 19/04/2018, «rancho» en Palenque). Muestra de ello es el reciente impulso para que las plantaciones comerciales de las empresas de la región puedan estar certificadas a través de la Mesa Redonda del Aceite de Palma Sustentable (RSPO, por sus siglas en inglés).

Las afirmaciones que abogan por una producción sustentable provienen de quienes manejan las plantaciones comerciales de las empresas y de algunos de los productores de pequeña escala. Estos discursos respaldan los beneficios ambientales de las plantaciones, así como las buenas prácticas de las plantas extractoras en una lógica de ser más amigables con la naturaleza. Casos contrastantes se pueden hallar en relatos de productores de pequeña escala quienes reflexionan sobre las contradicciones en torno a la idea de la palma como «buena para la tierra». Muchos de los campesinos-palmeros de la región no han escuchado o no tienen idea sobre «producción sustentable de la palma», esta visión es más conocida entre trabajadores de las empresas y algunos productores de la planicie.

La intención de estipular que es posible una "producción sustentable de la palma de aceite» es originada por presiones a nivel internacional, sobre todo de campañas de organizaciones ambientalistas que generaron una reacción en el sector empresarial (Pye, 2010), lo cual terminó por empujar iniciativas internacionales como la RSPO (Overbeek, Kröger, y Gerber, 2012). Un papel secundario lo han tomado los consumidores de los productos finales, quienes también han manifestado su rechazo al aceite vegetal de palma aceitera (Trejo, 2018). 
Bajo estos términos, la producción sustentable del aceite de palma es un asunto de política industrial, originada por discursos sobre sostenibilidad y conservación ambiental desde casos emblemáticos como el de Indonesia. Tal como lo indica Bissonnette (2012), los actores del agronegocio de palma de aceite constantemente moldean sus discursos para incorporar nuevas modalidades de conocimiento, en este caso conocimientos y discursos ambientales. Un discurso que, como muestra este estudio, aún no ha permeado en las visiones de los campesinos-palmeros.

\section{Discusión}

En los apartados anteriores se describen los cambios en las recientes dos décadas, los cuales coinciden con la presencia de la palma aceitera en la región. En relación con este periodo, se recapitulan los sucesos en la vida de los campesinos-palmeros en distintos ámbitos: agrícola, productivo, económico, organizativo y comercial. Esto nos coloca en el presente (2018) para entender cómo se ha construido un escenario de coexistencia entre el nuevo cultivo -y todo su circuito de comercio y procesamiento- y el sostenimiento de la vida familiar y comunitaria del sujeto colectivo en cuestión. Coexistencia que tiene que entenderse en un escenario de producción y reproducción de relaciones de poder, que en el corto plazo pueden ofrecer ventajas económicas y sociales a los campesinos-palmeros pero que a largo plazo «suponen su subordinación a los intereses de determinadas élites» (Castellanos, 2018:30).

El caso de Palenque devela una serie de intervenciones en territorios agrícolas que históricamente han sido objeto de políticas públicas que han buscado modernizar al campo mexicano. Diversas actividades agropecuarias fueron desarrolladas en las mismas zonas donde ahora la palma aceitera se expande. Tal es el caso de la ganaderización ocurrida en Palenque a mediados del siglo $X X$, siendo este uno de los municipios de Chiapas donde la ganaderización fue muy acelerada y llegó al límite de su potencial de expansión en la década de 1990 (Villafuerte et al., 1997:98-99). ${ }^{17}$ La ganaderización de la región produjo otra escalada de reconfiguraciones en las relaciones sociales (como la transculturación de la población indígena y cambios en la tenencia de la tierra, entre otras), aspectos fundamentales para entender cómo, en su momento, la ganadería se

\footnotetext{
17 Sobre el proceso de ganaderización véase Bobrow-Strain (2015:161-173).
} 
territorializó en Palenque. Con lo anterior no pretendemos argumentar que la sucesión de actividades productivas, o su empalme, haya sido pensada para que le abrieran el paso (intencionalmente) a la palma. Sin embargo, es importante recapitular las actividades que han moldeado los territorios agrícolas de Palenque en su historia reciente, así como las reconfiguraciones que estas generan en las relaciones socioambientales.

En las recientes décadas la palma aceitera ha sido el cultivo comercial más destacado en la zona de Palenque, esto si se considera la relativa importancia de otros cultivos como el hule, mientras que el maíz es cultivado en gran proporción para el autoconsumo y es visto, en algunos ámbitos, como un cultivo con poca importancia comercial.

Con la llegada de las plantaciones comerciales de palma aceitera, las cualidades que permiten al campesino ser artífice de sus propios medios de vida sufren una oleada más de reconfiguración, y se mantiene latente el cambio paulatino donde estos campesinos pasarían a ser simples productores de cultivos agroindustriales. La presencia de la palma ha decantado en que algunos de quienes la cultivan busquen mantenerse en el negocio dados los buenos resultados económicos, los cuales se traducen en estabilidad económica.

El caso estudiado nos devela parte de lo que Antonio Castellanos (2015) ya había detallado: el Estado mexicano (con sus interrelaciones con poblaciones locales) ha tenido una función como impulsor clave de la producción de aceite de palma en el sureste mexicano. Diversos agentes gubernamentales llegaron directamente a los ejidos de Palenque para hacer propaganda acerca de los beneficios económicos y productivos de la palma aceitera. Así se tejieron las vías principales para que pudiera insertarse en los paisajes agrícolas de Palenque y sus alrededores. Además, el acercamiento empírico nos indica una clara influencia del Estado mexicano para la llegada de las empresas extractoras a la región, lo que resuena con el estudio hecho por Castellanos donde destaca el proyecto gubernamental que favoreció al «sector privado y de aquellos con más recursos» (Castellanos, 2018:29).

A partir de la premisa de que los procesos globales impactan de manera diferenciada en ámbitos locales (Linares et al., 2018), podemos referir que, en el caso específico de Palenque, encontramos información empírica que nos explica el avance de la producción de palma a través de las intermediaciones entre el campesinado y el Estado mexicano. Pero, por otro lado, hay campesinos-palmeros que señalan varias debilidades en el apoyo otorgado por el gobierno, empezando por información inadecuada para entender las implicaciones ambientales de la producción de aceite de palma en sus parcelas, así como falta 
de seguimiento después de la entrega de los primeros insumos, seguido de la especulación sobre los tiempos en los que se establecerían las comercializadoras y procesadoras en la zona.

De igual forma, el caso esboza cómo los impulsos externos hallan cabida en la agenda local y cómo se entretejen diversas historias de vida para sumarse al impulso de un cultivo externo, y cómo, también, las relaciones socioeconómicas y ambientales suceden en los espacios de producción agrícola, comercio, transporte, extracción y distribución del aceite de palma. Además, se gestan procesos organizativos que implican relaciones intercomunitarias y con diversos agentes gubernamentales y empresariales, dinámicas a las cuales los campesinos de Palenque se han adaptado para cultivar para la agroindustria.

Aparentemente los territorios biofísicos (lo material) siguen con pocos cambios (como el hecho de la poca atribución que se le puede hacer a la palma en cuanto al desmonte de selva y acahuales) pero, en aspectos inmateriales (simbólicos) la palma llegó a reforzar procesos como la transculturación, una separación social ( entre «lo indígena» y «lo mestizo»), la dependencia de paquetes tecnológicos y la especialización en el ámbito agrícola (ser palmeros, ser productores, ser cortadores, ser cargadores, ser jornaleros, ser empresarios, ser ganaderos, etc.). Procesos que tienen una fuerte orientación a la modernización productiva afín a la agroindustrialización de los territorios campesinos.

\section{Conclusiones}

No es fácil identificar una sola vía de decisiones que llevaron a los productores de pequeña escala a sumarse a la implementación de plantaciones comerciales de palma aceitera. Dentro del alcance de esta investigación, identificamos que una gran proporción de los productores de pequeña escala decidieron involucrarse en la producción del fruto de la palma a raíz de las acciones propagandísticas de diversos agentes gubernamentales. Dicha tendencia fue el detonante para el establecimiento de plantaciones en la región de Palenque.

Los cambios en las territorialidades locales en relación a la expansión agroindustrial de la palma aceitera es lo que nos ha llevado a decir que esta ha incidido en diversos ámbitos de la vida de los campesinos. Para distinguir cómo se dio este proceso, en primera instancia, se debe reconocer especial protagonismo de quienes se involucran en la producción del fruto de la palma. Pero, en 
un segundo plano, también advertir que, quienes no están involucrados directamente han observado y tienen sus propios relatos e imaginarios en cuanto a la expansión de la palma en el territorio en el que viven. Esto nos señala que los cambios culturales que nos permiten hablar de campesinos-palmeros no se restringen a estos, sino que hay efectos del sistema agroindustrial sobre las vidas de otras familias en el territorio.

De acuerdo con lo analizado en estas páginas, el caso de la palma aceitera en Palenque como cultivo agroindustrial nos devela que su producción aún no supera las contradicciones entre beneficios o perjuicios ambientales. Es una actividad económica que comienza a desarrollar ciertos parámetros de control y «responsabilidad» ambiental como indicadores de producción sustentable, tanto en plantaciones como en plantas extractoras. Mientras que los beneficios económicos para los campesinos-palmeros y la economía local no afrontan sustantivamente temas como la erosión cultural, la desestructuración de tejidos comunitarios o la degradación de paisajes agrícolas.

\section{Agradecimientos}

Agradecemos a todas las personas de comunidades rurales de Palenque por la información y la confianza depositada. Así como a las personas revisoras, quienes atinadamente aportaron a mejorar la estructura y la redacción de este artículo. La realización de esta investigación fue posible gracias a la beca otorgada por el Consejo Nacional de Ciencia y Tecnología (CONACYT), durante los estudios de maestría del primer autor.

\section{Bibliografía citada}

Alimonda, Héctor. (2017). En clave de sur: la Ecología Política Latinoamericana y el pensamiento crítico. En Héctor Alimonda, Catalina Toro y Facundo Martín (coords.), Ecología política latinoamericana. Pensamiento crítico, diferencia latinoamericana y rearticulación epistémica (pp. 33-50). Buenos Aires: Consejo Latinoamericano de Ciencias Sociales/Universidad Autónoma Metropolitana.

Ávila Romero, Agustín y Jadson Albuquerque. (2018). Impactos socioambientales del cultivo de palma africana: los casos mexicano y brasileño. Economía y Sociedad, 23(53), pp. 63-83, doi.org/10.15359/eys.23-53.4 
Ávila Romero, León, Agustín Ávila y José Luis Sulvarán. (2017). The Myth Behind Sustainable African Crop. Socio-Environmental Impacts of Palm Oil in Chiapas, México. International Journal of Ecology \& Development, 32(4).

Bissonnette, Jean Francoise. (2012). Envisioning agribusiness: Land, labour and value in a time of oil palm expansion in Indonesia. Canadá: University of Toronto.

Bobrow-Strain, Aaron. (2015). Enemigos íntimos. Terratenientes, poder y violencia en Chiapas. México: Centro de Investigaciones Multidisciplinarias sobre Chiapas y la Frontera Sur-Universidad Nacional Autónoma de México/Consejo Estatal para las Culturas y las Artes/Universidad Autónoma de Chiapas/Universidad de Ciencias y Artes de Chiapas/Consejo de Ciencia y Tecnología en el Estado de Chiapas.

Cajas-Castillo, José O., Ana J. Cóbar-Carranza, Rafael C. Ávila-Santa Cruz, Cristian KrakerCastañeda y Juan M. Quiñónez-Guzmán. (2015). Diversidad de aves de sotobosque en bosques tropicales, áreas de renegeración natural y cultivos de palma africana en humedales del Lago de Izabal, Guatemala. Ornitología Neotropical, 26(1), pp. 1-12.

Cano Castellanos, Ingreet. J. (2014). Entre sueños agrarios y discurso ecologista. Las encrucijadas contemporáneas de la colonización de la Selva Lacandona (Chiapas, México). Entre Diversidades-Revista de Ciencias Sociales y Humanidades, (2), pp.101-143, doi: doi.org/10.31644/ED.2.2014.a04

Castellanos Navarrete, Antonio. (2015). Illusions, hunger and vices: smallholders, environmentalism and the green agrarian question in Chiapas'biofuel rush. Wageningen: Wageningen University.

Castellanos Navarrete, Antonio. (2018). Palma de aceite en tierras campesinas : la política de las transformaciones territoriales en Chiapas, México. Revista Pueblos y fronteras digital, 13, pp. 1-43, doi.org/10.22201/cimsur.18704115e.2018.v13.357

Castellanos Navarrete, Antonio y Kees Jansen. (2017). Why do smallholders plant biofuel crops? The "politics of consent" in Mexico. Geoforum, 87, october, pp. 15-27, doi. org/10.1016/j.geoforum.2017.09.019

Durand Smith Leticia, Fernanda Figueroa y Mauricio Guzmán. (2011). La ecología política en México ¿ Dónde estamos y para dónde vamos? Political Ecology in Mexico: ¿Where are we and where are we heading to ? Estudios Sociales, 19(37), pp. 282-307.

Escobar, Arturo. (2010). Territorios de diferencia: Lugar, movimientos, vida, redes. Popayán, Colombia: Envión Editores.

Fenner, Justus. (2015). La llegada al Sur. La controvertida historia de los deslindes de terrenos baldíos en Chiapas, México, en su contexto internacional y nacional, 1881-1917. San Cristóbal de Las Casas, Chiapas, México: CIMSUR/CONACULTA: CONECULTA/UNACH/UNICACH/COCYTECH.

Fletes Ocón, Héctor y Alessandro Bonanno. (2015). Respuestas a la crisis de la globalización neoliberal: intervención del Estado en la producción de aceite de palma en Chiapas, México. Carta Económica Regional, 27(116), pp. 5-35.

Friedmann, Harriet. (1982). The Political Economy of Food: The Rise and Fall of the Postwar International Food Order. The American Journal of Sociology, 88, pp. 29-57. 
Gobierno Constitucional del estado de Chiapas. (1991). La zona del Soconusco como potencial para el establecimiento del cultivo de la palma africana. Chiapas: Gobierno Constitucional del estado de Chiapas/Secretaría de Desarrollo Rural- Delegación 08.

Haesbaert, Rogério. (2011). El mito de la desterritorialización. Del "fin de los territorios" a la multiterritorialidad. México: Siglo XXI.

Haesbaert, Rogério. (2013). Del mito de la desterritorialización a la multiterritorialidad. Cultura y Representaciones Sociales, 8(15), pp. 9-42. Disponible en: http://www. scielo.org.mx/scielo.php?script=sci_arttext\&pid=S2007-81102013000200001\&ln$\mathrm{g}=\mathrm{es} \& \mathrm{t}$.ng=es (consulta: 28/03/2019).

Leff, Enrique. (2017). Las relaciones de poder del conocimiento en el campo de la ecología política. Ambiente \& Sociedade, XX(3), pp. 229-262.

Linares Bravo, Bárbara, Emma Zapata Martelo, Austreberta Nazar-Beutelspacher y Blanca Suárez-San Román. (2018). Reconversión productiva a palma de aceite en el Valle del Tulijá, Chiapas, México. Impacto diferenciado por género. Agricultura, sociedad y desarrollo, 15(4), pp. 487-506.

Martínez-Alier, Joan. (1997). Conflictos de distribución ecológica. Revista Andina, (1), pp. pp. 41-76.

McMichael, Philip. (1994). The Global Restructuring of Agro-Food Systems. London: Cornell University Press, Ithaca.

Mier y Terán Giménez Cacho, Mateo. (2016). Soybean agri-food systems dynamics and the diversity of farming styles on the agricultural frontier in Mato Grosso, Brazil. The Journal of Peasant Studies, 43(2), pp. 419-441, doi.org/10.1080/03066150.2015.101 6917

Neiman, Guillermo y Germán Quaranta. (2006). Los estudios de caso en la investigación sociológica. En Irene Vasilachis de Gialdino (ed.), Estrategias de investigación cualitativa (pp. 213-238). España: Gedisa.

Oliveira, Gustavo y Susanna Hecht. (2016). Sacred groves, sacrifice zones and soy production: globalization, intensification and neo-nature in South America. The Journal of Peasant Studies, 43(2), pp. 251-285, doi 10.1080/03066150.2016.1146705

Overbeek, Winfridus, Markus Kröger, y Julien-Francois Gerber. (2012). Una panorámica de las plantaciones industriales de árboles en países del Sur. Informe EJOLT (Vol. 3). Disponible en: http://www.ejolt.org/wordpress/wp-content/ uploads/2012/09/120913_EJOLT3-ESP-Low.pdf

Pérez-Brignoli, Héctor. (2017). Aculturación, transculturación, mestizaje: metáforas y espejos en la historiografía latinoamericana. Cuadernos de Literatura, XXI(41), pp. 96-113, doi.org/10.11144/Javeriana.cl21-41.atmm

Pye, Oliver. (2010). The biofuel connection - transnational activism and the palm oil boom. The Journal of Peasant Studies, 37(4), pp. 851-874, doi.org/10.1080/03066150.2010 .512461

Ramírez Velázquez, Blanca y Liliana López Levi. (2015). Espacio, paisaje, región, territorio y lugar: la diversidad en el pensamiento contemporáneo. México: UNAM, Instituto de Geografía/ UAM-Xochimilco. 
Ramírez Zamora, Rosalío.(1991). La palma africana; una alternativa para el trópico húmedo. Acapetahua, Chiapas. México: Secretaría de Agricultura y Recursos Hidráulicos; Instituto Nacional de Investigaciones Forestales y Agropecuarias/ Centro de Investigaciones Forestales y Agropecuarias de Chiapas.

Rangel, Francisco y Héctor Fletes Ocón. (2010). Pequeños productores, biocombustibles y guerras por el espacio. La sustentabilidad agrícola cuesta arriba. Elementos para el análisis desde el Soconusco Chiapaneco. En Héctor Fletes Ocón (ed.), Pequeños productores y vulnerabilidad global agroalimentaria (pp. 146-165). Chiapas: Red de Investigación Socioeconómica en Hortalizas, Frutas y Flores/ Universidad Autónoma de Chiapas.

Robbins, Paul. (2004). Political Ecology. A critical introduction. Arizona:Blackwell Publishing.

Santacruz de León, Eugenio y Victor Palacio Muñoz. (2018). Public policies for oil palm cultivation: the case of the Soconusco region, Chiapas, Mexico. Revista de Geografía Agrícola, 60(82).

Servicio de Información Agroalimentaria y Pesquera (SIAP). (2019). Anuario Estadístico de la Producción Agrícola. Disponible en https://nube.siap.gob. $\mathrm{mx} /$ cierreagricola/ (consulta: 06/07/2019).

Stake, Robert. (1998). Investigación con estudio de casos. Madrid, España: Morata. The World Bank. (2011). The World Bank Group Framework and IFC Strategy for Engagement in the Palm Oil Sector. Informe. Washington DC. USA: The World Bank and International Finance Corporation.

Trejo Sánchez, Enrique de Jesús. (2018). Reestructuración productiva y gobernanza en la cadena de palma de aceite en la microrregión costera de Chiapas de 1990 - 2015. Tesis de doctorado inédita, México: UNACH.

Villafuerte Solís, Daniel, Salvador Meza, Gabriel Ascencio, María del Carmen García Aguilar, Jesús Morales, Miguel Lisbona y Carolina Rivera. (2002). La tierra en Chiapas. Viejos problemas nuevos. México: Fondo de Cultura Económica.

Villafuerte, Daniel, María del Carmen García y Salvador Meza, S. (1997). La cuestión ganadera y la deforestación. Viejos y nuevos problemas en el trópico y Chiapas. Tuxtla Gutiérrez, Chiapas: Gobierno del Estado de Chiapas/ UNICACH.

Vos, Jan de. (1988). Oro Verde. La conquista de la Selva Lacandona por los madereros tabasqueños, 1822-1949. México, D.F.: FCE.

Washbrook, Sarah. (2018). La producción de la modernidad en México. Fuerza de trabajo, raza y Estado en Chiapas, 1876-1914. Chiapas: CIMSUR- UNAM.

\section{Cómo citar este artículo:}

Rodríguez Méndez, José Luis y Mateo Mier y Terán Giménez-Cacho. (2020). Expansión de la producción de aceite de palma en territorios campesinos, el caso de Palenque, Chiapas (1996-2018). Revista Pueblos y fronteras digital. 15, pp. 1-28, doi: 10.22201/cimsur.18704115e.2020.v15.445 